Check for updates

Cite this: Nanoscale Adv., 2019, 1, 1165

\title{
Platinum single-atom adsorption on graphene: a density functional theory study $\dagger$
}

\author{
Sasfan Arman Wella, (DD ab Yuji Hamamoto, (D) ac Suprijadi, (DD b \\ Yoshitada Morikawa (D) acd and Ikutaro Hamada (D) *ac
}

\begin{abstract}
Single-atom catalysis, which utilizes single atoms as active sites, is one of the most promising ways to enhance the catalytic activity and to reduce the amount of precious metals used. Platinum atoms deposited on graphene are reported to show enhanced catalytic activity for some chemical reactions, e.g. methanol oxidation in direct methanol fuel cells. However, the precise atomic structure, the key to understand the origin of the improved catalytic activity, is yet to be clarified. Here, we present a computational study to investigate the structure of platinum adsorbed on graphene with special emphasis on the edges of graphene nanoribbons. By means of density functional theory based thermodynamics, we find that single platinum atoms preferentially adsorb on the substitutional carbon sites at the hydrogen terminated graphene edge. The structures are further corroborated by the core level shift calculations. Large positive core level shifts indicate the strong interaction between single Pt atoms and graphene. The atomistic insight obtained in this study will be a basis for further investigation of the activity of single-atom catalysts based on platinum and graphene related materials.
\end{abstract}

Received 23rd September 2018

Accepted 8th December 2018

DOI: $10.1039 / c 8 n a 00236 c$

rsc.li/nanoscale-advances

\section{Introduction}

High-performance electrocatalysts are highly desired for electrochemical energy conversion devices, such as photovoltaic cells and fuel cells. Platinum (Pt) is widely used as an electrocatalyst, as it exhibits high catalytic activity not only for hydrogen oxidation but also for oxygen reduction at low temperatures. ${ }^{1,2}$ Nevertheless, there is still an urgent need to address the high cost of Pt and to search for alternative catalysts, which use small amounts of Pt or no Pt with earth-abundant materials. Tremendous efforts have been devoted to achieve these goals, including the use of non-precious metals for Pt-metal alloys, ${ }^{3-9}$ or the use of noble metal-free catalysts. ${ }^{10-13}$ Particularly interesting are Pt clusters supported by graphitic materials, such as carbon black, carbon nanotubes, and graphene, which have been extensively studied both experimentally, ${ }^{14-20}$ and theoretically. ${ }^{21-34} \mathrm{~A}$ better catalytic

${ }^{a}$ Department of Precision Science and Technology, Graduate School of Engineering, Osaka University, 2-1 Yamada-oka, Suita, Osaka 565-0871, Japan. E-mail: ihamada@prec.eng.osaka-u.ac.jp

${ }^{b}$ Department of Physics, Faculty of Mathematics and Natural Sciences, Institut Teknologi Bandung, Jalan Ganesha 10, Bandung 40132, Indonesia

'Element Strategy Initiative for Catalyst and Batteries, Kyoto University, Katsura, Kyoto 615-8520, Japan

${ }^{d}$ Research Center for Ultra-Precision Science and Technology, Graduate School of Engineering, Osaka University, 2-1 Yamada-oka, Suita, Osaka 565-0871, Japan

$\dagger$ Electronic supplementary information (ESI) available: Functional dependence study on the stability of graphene nanoribbons and electronic structure analysis of single platinum atoms at the graphene edge. See DOI: 10.1039/c8na00236c activity of small Pt clusters supported by graphene sheets has been demonstrated experimentally. ${ }^{16,17}$ These results lead to the hypothesis that downsizing the Pt clusters to single atoms can enhance the catalytic activity. Recently, Sun et al. have successfully deposited single Pt atoms on graphene nanosheets using atomic layer deposition and then demonstrated a significant improvement of the catalytic activity for the methanol oxidation reaction. ${ }^{35}$ Cheng et al. also showed that a Pt single-atom catalyst deposited on nitrogen doped graphene nanosheets exhibits enhanced catalytic activity for the hydrogen evolution reaction. ${ }^{36}$ The vacancies usually formed during the preparation are expected to have strong interactions with the Pt atoms as demonstrated in several theoretical studies. ${ }^{23,31,32}$ Back et al. have predicted the great potential of a single atom catalyst supported on defective graphene for $\mathrm{CO}_{2}$ electroreduction applications. ${ }^{37}$ However, the dispersion of the single Pt atoms on graphene is limited to the number of point defects. On the other hand, the graphene edge might offer more space for depositing single Pt atoms. Kong et al. have theoretically investigated Pt single-atom adsorption at the edges of graphite nanofibers and found that the atoms are tightly bound to the edges due to the existence of active dangling bonds. ${ }^{38}$ By employing transmission electron microscopy, the structure and dynamics of $\mathrm{Au},{ }^{39} \mathrm{Fe},{ }^{40} \mathrm{Cu},{ }^{41}$ and $\mathrm{Pt}^{41,42}$ atoms at the edges have been studied. Some experimental studies also observed that Pt nano-clusters at the graphene edges are stable at high-temperatures. ${ }^{43-45}$ However, the adsorption state and the catalytic activities of single Pt atoms are not yet fully understood. 
In this work, we investigate Pt single-atom adsorption on graphene by means of density functional theory (DFT) that includes van der Waals forces. We perform systematic calculations to determine the adsorption state of single Pt atoms on graphene, including defective graphene structures and graphene edges. Special emphasis is devoted to the graphene edges, as they are abundant under realistic conditions. We consider both zigzag and armchair edges, including the dependence on hydrogen termination. We examine their stability by taking into account the environmental effects, via DFT based thermodynamics. ${ }^{46}$ We find that single Pt atoms adsorb preferentially at the edge rather than on graphene, and the substitutional carbon site is the most stable one under the conditions relevant to experiments. Furthermore, the core level shift (CLS) of Pt atoms is calculated for each structure, which is used to validate the predicted structure against the experiment.

\section{Computational details}

All the DFT calculations are performed by using STATE $\operatorname{code} \mathrm{e}^{47,48}$ with ultrasoft pseudopotentials. ${ }^{49}$ A plane-wave basis set is used to expand wave functions and charge density with cutoff energies of 36 Ry and 400 Ry, respectively. We use rev-vdW-DF2 (ref. 50) exchange correlation functional as implemented ${ }^{51}$ in the code with an efficient algorithm..$^{52,53}$ Pseudopotentials are generated using the Perdew-Burke-Ernzerhof (PBE) ${ }^{54}$ functional and the use of PBE pseudopotentials in rev-vdW-DF2 calculations is validated in ref. 55 . We use a $(6 \times 6)$ supercell to simulate Pt single-atom adsorption on pristine (GR), monocarbon vacancy $\left(V_{1}\right)$, and di-carbon vacancy $\left(V_{2}\right)$ graphene structures. We introduce the 7575 membered rings of graphene as a boundary between zigzag and armchair as observed in ref. 56 , denoted as grain-boundary graphene (GB-GR). We place the GB-GR in a $17.23 \AA \times 14.03 \AA$ rectangular unit cell. We also construct disorder graphene (DisGR) reported in ref. 57, and place it in a $20.37 \AA \times 24.67 \AA$ rectangular cell. Brillouin zone integration is performed for all structures using the $\Gamma$-centered $4 \times 4 k$-point mesh for pristine, mono-carbon vacancy, and dicarbon vacancy graphenes; $2 \times 3 k$-point mesh for GB-GR; and the $\Gamma$-point for DisGR. To investigate the edge effect in the $\mathrm{Pt}$ adsorption, we employ graphene nanoribbons (GNRs) with zigzag ( $z \mathrm{GNR})$ and armchair $(a \mathrm{GNR})$ edges with different terminations, including non-hydrogenated, mono-hydrogenated, and di-hydrogenated ones. We follow the convention used in ref. 58, i.e., $z_{n}$ to denote $z$ GNR with $n$ hydrogen atoms at the edge carbon (C) site, and $a_{n}$ for $a$ GNR with $n$ hydrogen (H) atoms. Spin polarization is taken into account for all systems. $z$ GNR has the localized spin with ferromagnetic order along the edge and antiparallel orientation between the edges as reported in ref. 59 and 60, while such localized spin does not appear at the edge of $a$ GNR. GNRs are modeled using a periodic supercell along the edges, having the same configurations on both edges. Following the conventional notation, the width of GNR is specified by the number of zigzag chains and dimer lines for $z \mathrm{GNR}$ and $a \mathrm{GNR}$, respectively, and we use $5-z \mathrm{GNR}$ and $10-a \mathrm{GNR}$ in this work. We use supercells containing 4 hexagons for both $z \mathrm{GNR}$ and $a \mathrm{GNR}$, and the resulting supercells contain 40 carbon atoms. Supercells in the graphene plane directions correspond to $(4 \times 6 \sqrt{3})$ and $(2 \sqrt{3} \times 12)$ supercells of graphene for $z$ GNR and $a \mathrm{GNR}$, respectively, and graphene planes are separated by a vacuum of $\sim 15 \AA$ thickness. Vacuum thicknesses between edges are $16.48 \AA$ and $18.70 \AA$ for $z \mathrm{GNR}$ and $a \mathrm{GNR}$, respectively. Brillouin zone integration is performed using the $\Gamma$-centered 6 $\times 2 k$-point set for both $z \mathrm{GNR}$ and $a \mathrm{GNR}$. All the graphene based structures considered in this work are constructed using the lattice constant of graphene obtained using rev-vdW-DF2 $(2.46 \AA),{ }^{61}$ which is in good agreement with the experimental value for graphite $(2.4589 \pm 0.0005 \AA) .{ }^{62}$ The structures are fully relaxed until the forces acting on the atoms become smaller than $5.14 \times 10^{-2} \mathrm{eV}^{-1}\left(1 \times 10^{-3}\right.$ Hartree/Bohr $)$. The Pt 4 f CLS including the final state screening is calculated as the difference between the core level binding energy of Pt adsorbed GNR and bulk Pt. ${ }^{63,64}$

\section{Results and discussion}

\subsection{Pt single-atom adsorption on pristine and defective graphene structures}

We first consider pristine and defective graphene structures and adsorption of single Pt atoms on them as shown in Fig. 1. We calculate the binding energy defined by

$$
E_{\mathrm{b}}=E_{\mathrm{Pt} / \mathrm{G}}-E_{\mathrm{G}}-\mu_{\mathrm{Pt}},
$$

where $E_{\mathrm{Pt} / \mathrm{G}}, E_{\mathrm{G}}$, and $\mu_{\mathrm{Pt}}$ are the total energy of the adsorption system, the total energy of the substrate, and the chemical potential of Pt, respectively. We use the total energy of an isolated Pt atom for $\mu_{\mathrm{Pt}}$. In Table 1, we summarize the calculated binding energies of a single Pt atom on pristine and defective graphene structures. The binding energy for the pristine graphene is slightly larger than those reported in ref. 26 because of the exchange-correlation functionals used (see Table $\mathrm{S} 1$ in the $\mathrm{ESI} \dagger$ for the binding energies calculated using the $\mathrm{PBE}^{54}$ functional). The Pt single-atom adsorption on graphene with vacancy is significantly stable, because the Pt atom terminates
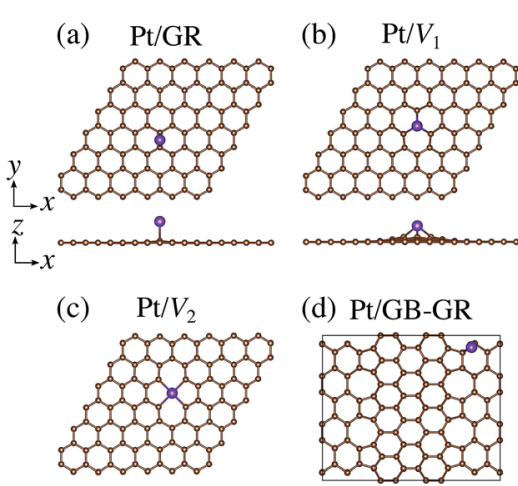

(d) $\mathrm{Pt} / \mathrm{GB}-\mathrm{GR}$

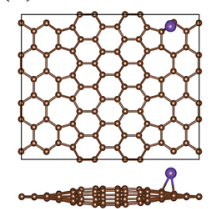

(e) Pt/DisGR

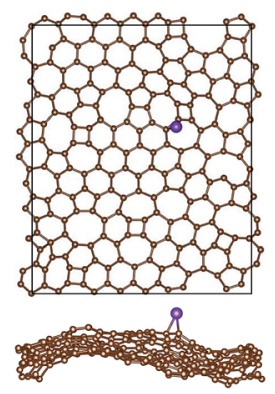

Fig. 1 Optimized structures of Pt single-atom adsorption on (a) pristine graphene (GR), (b) graphene with a carbon mono-vacancy $\left(V_{1}\right),(c)$ graphene with a carbon di-vacancy $\left(V_{2}\right),(d)$ grain-boundary graphene (GB-GR), and (e) disorder graphene (DisGR). 
Table 1 Binding energy $\left(E_{\mathrm{b}}\right)$ of Pt single-atom adsorption for several graphene based structures

\begin{tabular}{lc}
\hline Structure & $E_{\mathrm{b}} / \mathrm{eV}$ \\
\hline $\mathrm{Pt} / \mathrm{GR}$ & -1.97 \\
$\mathrm{Pt} / V_{1}$ & -7.70 \\
$\mathrm{Pt} / V_{2}$ & -7.57 \\
$\mathrm{Pt} / \mathrm{GB}-\mathrm{GR}$ & -2.55 \\
$\mathrm{Pt} /$ DisGR & -2.99
\end{tabular}

dangling bonds associated with the $\mathrm{C}$ vacancy. However, the energies necessary to create the $\mathrm{C}$ vacancies (formation energies for $\mathrm{C}$ vacancy with respect to the $\mathrm{C}$ atom in graphene) are 7.77 $\mathrm{eV}$ and $7.86 \mathrm{eV}$ for $V_{1}$ and $V_{2}$, respectively, and the effective binding energies are $+0.07 \mathrm{eV}$ and $+0.29 \mathrm{eV}$, respectively. Thus we conclude that the $\mathrm{Pt}$ single-atom adsorption at the $\mathrm{C}$ vacancy site is thermodynamically less favorable. The binding energies of a single Pt atom for GB-GR and DisGR are apparently much larger than that for pristine graphene. However, this is because GB-GR and DisGR are less stable and thus more reactive than the pristine one. They are less stable than pristine graphene by 0.17 and $0.39 \mathrm{eV}$ per $\mathrm{C}$ atom, respectively.

3.1.1 Pristine GNR. We then investigate the most stable GNR by calculating the formation energy defined by

$$
E_{\mathrm{form}}=\frac{1}{2 L}\left(E_{\mathrm{GNR}}-N_{\mathrm{C}} E_{\mathrm{GR}}-\frac{N_{\mathrm{H}}}{2} \mu_{\mathrm{H}_{2}}\right),
$$

where $E_{\mathrm{GNR}}, E_{\mathrm{GR}}$, and $\mu_{\mathrm{H}_{2}}$ are the total energy of GNR, total energy of $\mathrm{C}$ atoms in bulk graphene (total energy of graphene per atom), and chemical potential of $\mathrm{H}_{2}$ molecule, respectively; $N_{\mathrm{C}}\left(N_{\mathrm{H}}\right)$ is the number of $\mathrm{C}(\mathrm{H})$ atoms in GNR; and $L$ is the length of the unit cell along the edge. Fig. 2 shows the calculated formation energy as a function of $\mathrm{H}_{2}$ chemical potential. It is found that at high $\mathrm{H}_{2}$ chemical potential, $a \mathrm{GNR}\left(a_{2}\right)$ is stable, whereas $z$ GNRs are more stable at low $\mathrm{H}_{2}$ chemical potential, and non-hydrogenated GNR is unstable in a wide range of $\mathrm{H}_{2}$

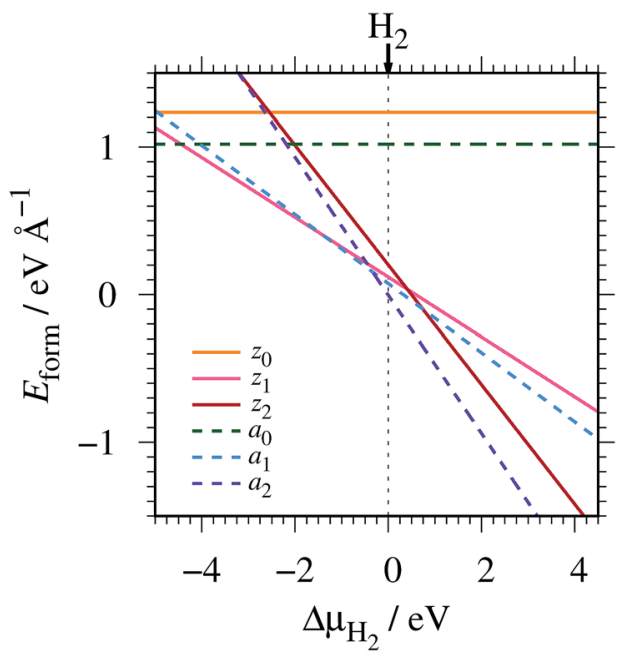

Fig. 2 Formation energy of GNR as a function of $\mathrm{H}_{2}$ chemical potential. $\Delta \mu_{\mathrm{H}_{2}}=\mu_{\mathrm{H}_{2}}-E_{\mathrm{H}_{2}}$, where $E_{\mathrm{H}_{2}}$ is the total energy of a gas-phase $\mathrm{H}_{2}$ molecule at $\mathrm{O} \mathrm{K}$. chemical potential, in good agreement with the previous study. ${ }^{58}$ The positions of the intersections differ from those reported in ref. 58, because the exchange-correlation functionals used are different (see Fig. S1 in the ESI $\dagger$ for comparison of the results obtained using rev-vdW-DF2 and PBE). We also confirm that the stability is insensitive to the GNR width (see Fig. S2 in the ESI†).

To compare the stability of the GNRs with the defective graphene structures, we calculate the formation energy per $\mathrm{C}$ atom (replace $2 L$ in eqn (2) with $N_{\mathrm{C}}$ ). At $\mu_{\mathrm{H}_{2}}=E_{\mathrm{H}_{2}}$, the formation energy per $\mathrm{C}$ atom of $z_{0}, z_{1}, z_{2}, a_{0}, a_{1}$, and $a_{2}$ are 0.61, 0.06, 0.10, $0.44,0.03$, and $\sim 0.00 \mathrm{eV}$, respectively. Thus, although most GNRs considered here are less stable than pristine graphene (i.e. most GNRs have positive $E_{\text {form }}$ at $\mu_{\mathrm{H}_{2}}=E_{\mathrm{H}_{2}}$ ), hydrogenated GNRs are more stable than defective graphene structures.

\subsection{Pt single-atom adsorption on $z$ GNRs}

We consider Pt single-atom adsorption on non-hydrogenated $z$ GNR $\left(z_{0}\right)$. We systematically construct the adsorption configurations with and without defects, and label them according to the Kröger-Vink notation, ${ }^{65}$ as shown in Fig. 3. We first consider the $\mathrm{Pt}$ single-atom adsorption at the top- and bridge-site of perfect $z_{0}$, denoted as $\mathrm{Pt}_{\mathrm{T}} @ z_{0}$ (Fig. 3(a)) and $\mathrm{Pt}_{\mathrm{B}} @ z_{0}$ (Fig. 3(b)), respectively. Second, we introduce a Stone-Wales (SW) defect at the edge of $z_{0}$, denoted as $z(57)_{0}$, and a Pt atom adsorbed at the long-bridge (LB) site of the SW defect, denoted as $\mathrm{Pt}_{\mathrm{LB}} @ z(57)_{0}$ (Fig. 3(c)). We introduce substitutional Pt with single edge carbon atoms $\left(\mathrm{C}_{\alpha}, \mathrm{C}_{\beta}\right)$ denoted as $\mathrm{Pt}_{\mathrm{C}_{\alpha}} @ z_{0}$ (Fig. 3(d)) and $\mathrm{Pt}_{\mathrm{C}_{\beta}} @ z_{0}$

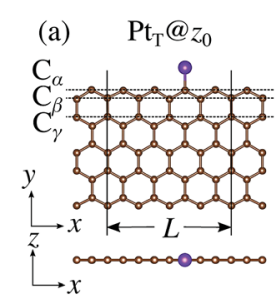

(b) $\quad \mathrm{Pt}_{\mathrm{B}} @ z_{0}$

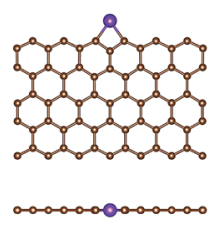

(e) $\quad \mathrm{Pt}_{\mathrm{C}_{\beta}} @ z_{0}$

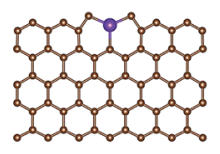

(f) $\quad \mathrm{Pt}_{\left(\mathrm{C}_{\alpha}\right)_{2}} @ z_{0}$

(d)

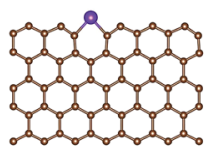

(g) $\mathrm{Pt}_{\mathrm{C}_{\alpha}} V_{\mathrm{C}_{\alpha}} @ z_{0}$

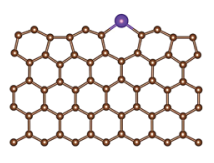

(h) $\quad \mathrm{Pt}_{\mathrm{C}_{\alpha} \mathrm{c}_{\beta}} @ z_{0}$

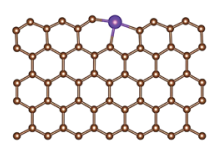

(i) $\quad \mathrm{Pt}_{\mathrm{C}_{\beta} \mathrm{C}_{\gamma}} @ z_{0}$

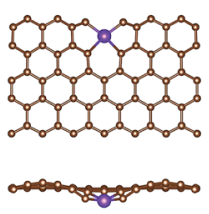

Fig. 3 Optimized structures of Pt single-atom adsorption at the edge of non-hydrogenated $z$ GNRs. Purple (brown) spheres represent $\mathrm{Pt}(\mathrm{H})$ atoms. The first, second, and third outermost carbon atoms are denoted as $C_{\alpha}, C_{\beta}$, and $C_{\gamma}$, respectively. $L$ indicates the periodicity of the ribbons along the edge direction. 
(Fig. 3(e)), respectively. We also consider two outermost carbon vacancies $\left(V_{\mathrm{C}}\right)$, and obtained the Pt substituted with two $V_{\mathrm{C}}$ 's (Pt $\left(\mathrm{C}_{\alpha}\right) @ z_{0}$, Fig. 3(f)) and a complex of a substitutional Pt and $V_{\mathrm{C}}$ $\left(\mathrm{Pt}_{\mathrm{C}_{\alpha}} V_{\mathrm{C}_{\alpha}} @ z_{0}\right.$, Fig. 3(g)). Finally we consider Pt configurations with a divacancy formed with the outermost and second outermost atoms and that with second and third outermost $\mathrm{C}$ atoms $\left(\mathrm{Pt}_{\mathrm{C}_{\alpha^{-}}}\right.$ ${ }_{\mathrm{C} \beta} @ z_{0}$ (Fig. 3(h)) and $\mathrm{Pt}_{\mathrm{C}_{\beta} \mathrm{C}_{\gamma}}$ @ $z_{0}$ (Fig. 3(i), respectively).

For the optimized structures with a single Pt atom at the edge of $z_{0}$, we calculate the Gibbs free energy defined by

$$
\Delta \Omega\left(\mu_{\mathrm{C}}\right)=E_{\mathrm{Pt} @ \mathrm{GNR}}-E_{\mathrm{ref}}-\mu_{\mathrm{Pt}}-\Delta N_{\mathrm{C}} \mu_{\mathrm{C}}
$$

where $E_{\mathrm{Pt} @ G N R}$ and $E_{\text {ref }}$ are the total energies of adsorbed and reference systems, respectively; $\Delta N_{\mathrm{C}}$ is the difference of the number of $\mathrm{C}$ atoms from the reference system; and $\mu_{\mathrm{Pt}}$ and $\mu_{\mathrm{C}}$ are the chemical potentials of $\mathrm{Pt}$ and $\mathrm{C}$, respectively. Here we choose pristine graphene as the reference $\left(E_{\mathrm{ref}}=E_{\mathrm{GR}} \times N_{\mathrm{C}}\right.$, where $N_{\mathrm{C}}$ is the number of $\mathrm{C}$ atoms in the perfect GNR). $\mu_{\mathrm{Pt}}$ is chosen to be the total energy of an isolated Pt atom $\left(E_{\mathrm{Pt}}\right)$ and $\mu_{\mathrm{C}}$ varies around the chemical potential of graphene. Fig. 4 shows the Gibbs free energy as a function of $\mathrm{C}$ chemical potential for nine structures considered in this work, and those at $\mu_{\mathrm{C}}=E_{\mathrm{GR}}$ (the system is in equilibrium with graphene) are summarized in Table 2. We can see that all the structures have large positive Gibbs free energies at $\mu_{\mathrm{C}}=E_{\mathrm{GR}}$, suggesting that Pt single-atom adsorption at the edges of $z_{0}$ is thermodynamically unstable. Among the Pt adsorption structures on $z_{0}, \mathrm{Pt}_{\mathrm{C}_{\alpha}} @ z_{0}$ is the most favorable configuration. However, the calculated CLS for $\mathrm{Pt}_{\mathrm{C}_{\alpha}}$ @ $z_{0}$ is too small compared with the experimental value of $(+2.0 \pm 0.4) \mathrm{eV},{ }^{42}$ suggesting that this adsorption configuration is unlikely. On the other hand, $\mathrm{Pt}_{\mathrm{C}_{\beta}} @ z_{0}$ and $\mathrm{Pt}_{\mathrm{C}_{\beta} \mathrm{C}_{\gamma}} @ z_{0}$ show a relatively large CLS of +1.44 and $+1.67 \mathrm{eV}$, respectively. However, their Gibbs free energies are significantly large, ruling out these configurations. Thus, we conclude that Pt single-atom adsorption on the non-hydrogenated GNR is unlikely under the equilibrium conditions.

We then investigate the stability of Pt single-atom adsorption on mono- and di-hydrogenated zGNRs $\left(z_{1}\right.$ and $\left.z_{2}\right)$. The

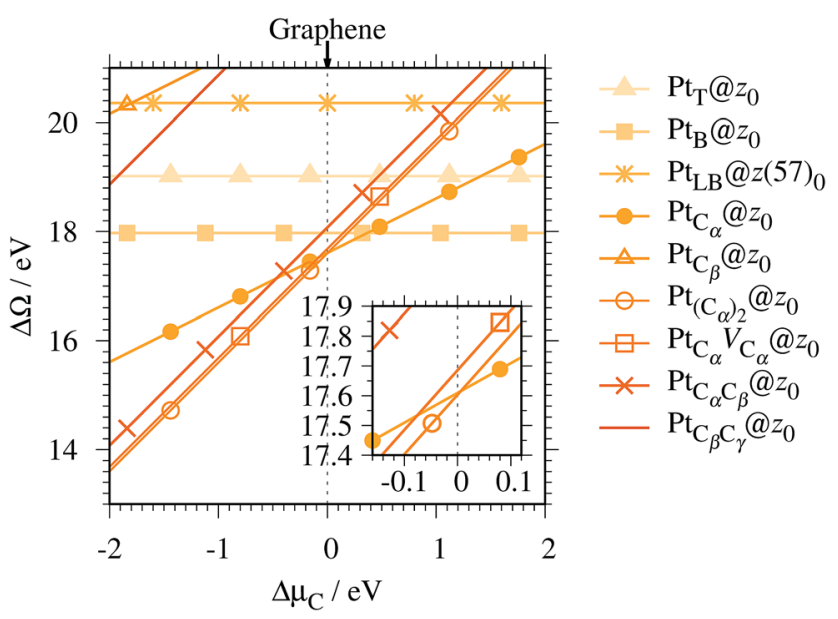

Fig. 4 The Gibbs free energy for the pristine GNRs as a function of $C$ chemical potentials. $\Delta \mu_{\mathrm{C}}=\mu_{\mathrm{C}}-E_{\mathrm{GR}}$ is the $\mathrm{C}$ chemical potential referenced to the total energy of graphene at $0 \mathrm{~K}\left(E_{\mathrm{GR}}\right)$.
Table 2 Gibbs free energy $(\Delta \Omega)$ and core level shift (CLS) of $\mathrm{Pt}$ atomazGNRs at $\mu_{\mathrm{C}}=E_{\mathrm{GR}}$ and $\mu_{\mathrm{H}}=E_{\mathrm{H}_{2}}$

\begin{tabular}{llllllll}
\hline & \multicolumn{3}{c}{$\Delta \Omega\left(E_{\mathrm{GR}}, E_{\mathrm{H}_{2}}\right) / \mathrm{eV}$} & & \multicolumn{3}{c}{$\mathrm{CLS} / \mathrm{eV}$} \\
\cline { 2 - 3 } \cline { 6 - 7 } & $z_{0}$ & $z_{1}$ & $z_{2}$ & & $z_{0}$ & $z_{1}$ & $z_{2}$ \\
\hline $\mathrm{Pt}_{\mathrm{T}}$ & +19.02 & +0.15 & +1.61 & & -0.56 & -0.93 & -1.30 \\
$\mathrm{Pt}_{\mathrm{B}}$ & +17.98 & +1.57 & +2.57 & & +0.44 & +0.35 & +0.35 \\
$\mathrm{Pt}_{\mathrm{LB}}{ }^{a}$ & +20.35 & +3.88 & +4.86 & & +0.44 & +0.44 & +0.29 \\
$\mathrm{Pt}_{\mathrm{C}_{\alpha}}$ & +17.61 & -0.25 & +1.92 & & +0.50 & +1.43 & +1.20 \\
$\mathrm{Pt}_{\mathrm{C}_{\beta}}$ & +22.16 & +1.89 & +0.51 & & +1.44 & +1.37 & +1.42 \\
$\mathrm{Pt}_{\left(\mathrm{C}_{\alpha}\right)}$ & +17.60 & +1.79 & +2.98 & & -0.29 & +0.24 & +0.48 \\
$\mathrm{Pt}_{\mathrm{C}_{\alpha} V_{\mathrm{C}_{\alpha}}}$ & +17.69 & +2.02 & +3.33 & & -0.42 & +0.54 & +0.88 \\
$\mathrm{Pt}_{\mathrm{C}_{\alpha} \mathrm{C}_{\beta}}$ & +18.08 & +0.14 & +1.62 & & +0.81 & +0.81 & +0.52 \\
$\mathrm{Pt}_{\mathrm{C}_{\beta} \mathrm{C}_{\gamma}}$ & +22.87 & +2.11 & +1.21 & & +1.67 & +0.82 & +1.11 \\
$\mathrm{Expt}$ & - & - & - & & & $+2.0 \pm 0.4$
\end{tabular}

${ }^{a} \mathrm{Pt}_{\mathrm{LB}}$ should be adsorbed at the edge of $z(57)_{n} \cdot{ }^{b}$ Taken from ref. 42.

structures are similar to those adopted for non-hydrogenated $z$ GNRs as shown in Fig. 5 and 6 for $z_{1}$ and $z_{2}$, respectively. We optimized all the structures and calculated the Gibbs free energy given by

$$
\Delta \Omega\left(\mu_{\mathrm{C}}, \mu_{\mathrm{H}_{2}}\right)=E_{\mathrm{Pt}} @_{\mathrm{GNR}}-E_{\mathrm{ref}}-\mu_{\mathrm{Pt}}-\Delta N_{\mathrm{C}} \mu_{\mathrm{C}}-\frac{\Delta N_{\mathrm{H}}}{2} \mu_{\mathrm{H}_{2}},
$$

where $E_{\mathrm{Pt} @ \mathrm{GNR}}\left(E_{\mathrm{ref}}\right)$ is the total energy of the adsorbed system (reference system), $\mu_{\mathrm{Pt}}=E_{\mathrm{Pt}}, \Delta N_{\mathrm{C}}$, and $\Delta N_{\mathrm{H}}$ are the difference of numbers of $\mathrm{C}$ and $\mathrm{H}$ atoms from the reference system, respectively, and $\mu_{\mathrm{C}}$ and $\mu_{\mathrm{H}_{2}}$ are chemical potentials of $\mathrm{C}$ atom and $\mathrm{H}_{2}$ molecule, respectively. As in the case of $z_{0}$, pristine graphene is

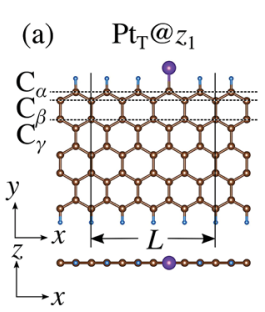

(b) $\quad \mathrm{Pt}_{\mathrm{B}} @ z_{1}$

(c) $\quad \mathrm{Pt} @ z(57)_{1}$

(d) $\quad \mathrm{Pt}_{\mathrm{C}_{\alpha}} @ z_{1}$
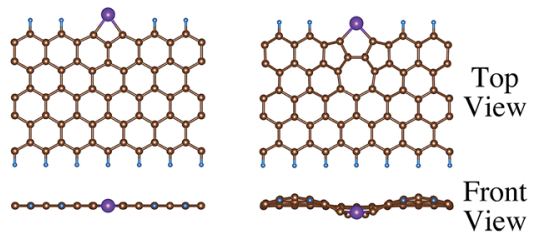

(e) $\quad \mathrm{Pt}_{\mathrm{C}_{\beta}} @ z_{1}$

(f) $\quad \mathrm{Pt}_{\left(\mathrm{C}_{\alpha}\right)_{2}} @ z_{1}$
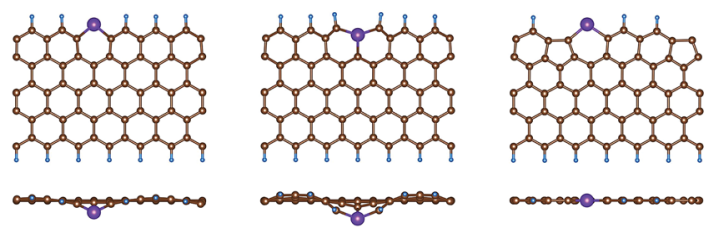

(g) $\mathrm{Pt}_{\mathrm{C}_{\alpha}} V_{\mathrm{C}_{\alpha}} @ z_{1}$

(h) $\quad \mathrm{Pt}_{\mathrm{C}_{\alpha} \mathrm{C}_{\beta}} @ z_{1}$

(i) $\mathrm{Pt}_{\mathrm{C}_{\beta} \mathrm{C}_{\gamma}} @ z_{1}$
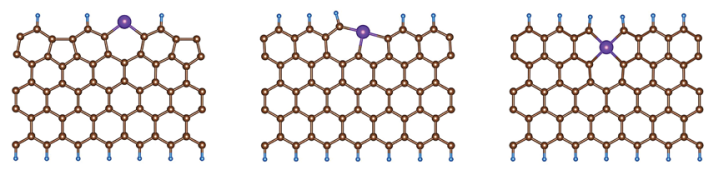

Fig. 5 Optimized structures of Pt single-atom adsorption at the edges of mono-hydrogenated $z$ GNRs. Purple, brown, and light blue spheres represent $\mathrm{Pt}, \mathrm{C}$, and $\mathrm{H}$ atoms, respectively. The first, second, and third outermost $C$ atoms are denoted as $C_{\alpha,} C_{\beta}$, and $C_{\gamma}$, respectively. $L$ indicates the periodicity of the ribbons along the edge direction. 


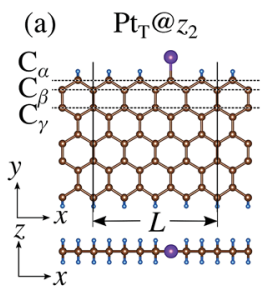

(b) $\quad \mathrm{Pt}_{\mathrm{B}} @ z_{2}$

(c) $\mathrm{Pt}_{\mathrm{LB}} @ z(57)_{2}$

(d) $\quad \mathrm{Pt}_{\mathrm{C}_{\alpha}} @ z_{2}$
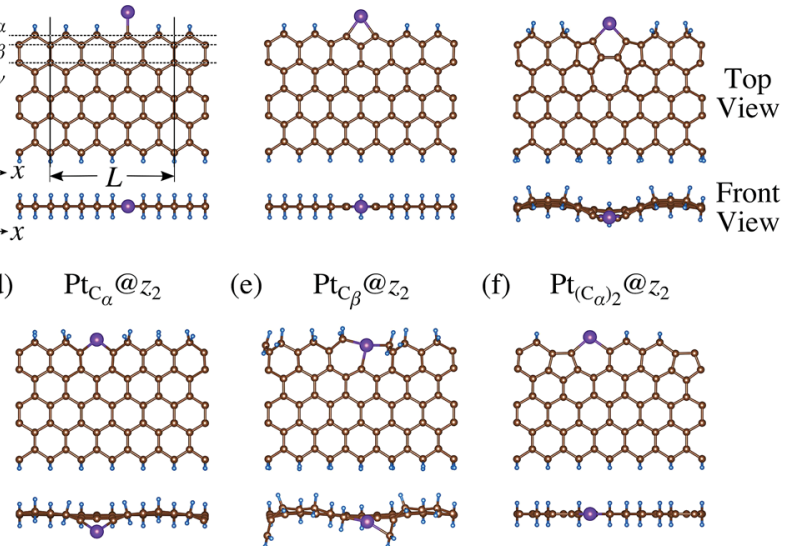

(e) $\quad \mathrm{Pt}_{\mathrm{C}_{\beta}} @ z_{2}$

(f) $\quad \mathrm{Pt}_{\left(\mathrm{C}_{\alpha}\right)_{2}} @ z_{2}$
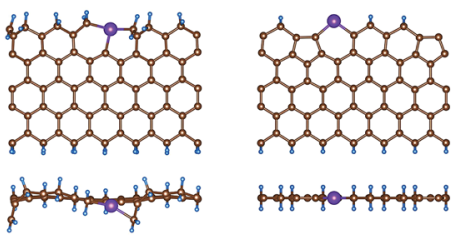

का की

(g) $\mathrm{Pt}_{\mathrm{C}_{\alpha}} V_{\mathrm{C}_{\alpha}} @ z_{2}$

(h) $\quad \mathrm{Pt}_{\mathrm{C}_{\alpha} \mathrm{C}_{\beta}} @ z_{2}$

(i) $\quad \mathrm{Pt}_{\mathrm{C}_{\beta} \mathrm{C}_{\gamma}} @ z_{2}$
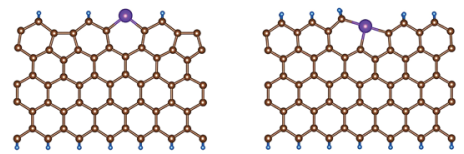

posfooffoto of of

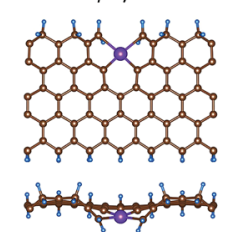

Fig. 6 Optimized structures of Pt single-atom adsorption at the edges of di-hydrogenated $z$ GNRs. Purple, brown, and light blue spheres represent $\mathrm{Pt}, \mathrm{C}$, and $\mathrm{H}$ atoms, respectively. The first, second, and third outermost $C$ atoms are denoted as $C_{\alpha}, C_{\beta}$, and $C_{\gamma}$, respectively. $L$ indicates the periodicity of the ribbons along the edge direction.

chosen as the reference. We calculate $\Delta \Omega$ for $z_{0}, z_{1}$, and $z_{2}$ as a function of $\mu_{\mathrm{H}_{2}}$ and $\mu_{\mathrm{C}}$ and generate the phase diagram as shown in Fig. 7. We also calculate $\Delta \Omega$ at $\mu_{\mathrm{C}}=E_{\mathrm{GR}}$ and $\mu_{\mathrm{H}_{2}}=E_{\mathrm{H}_{2}}$ for the structures considered, where $E_{\mathrm{H}_{2}}$ is the total energy of an isolated $\mathrm{H}_{2}$ molecule. The results are summarized in Table 2 along with the calculated CLSs for each structure. As expected, single Pt atom adsorbed non-hydrogenated $z$ GNR is unstable and does not appear in the phase diagram. We find that $\mathrm{Pt}_{\mathrm{C}_{\alpha}} @ z_{1}$ only exhibits negative $\Delta \Omega$ at $\mu_{\mathrm{C}}=E_{\mathrm{GR}}$ and $\mu_{\mathrm{H}_{2}}=E_{\mathrm{H}_{2}}$, suggesting that this structure is thermodynamically stable under these conditions. Furthermore, the calculated CLS for this structure is in reasonable agreement with the experiment. Among the hydrogenated GNR structures, calculated CLSs for $\mathrm{Pt}_{\mathrm{C}_{\beta}} @ z_{1}$ and $\mathrm{Pt}_{\mathrm{C}_{\alpha}} @ z_{2}$ are also reasonable. However, they show positive $\Delta \Omega$ 's, implying that these structures are less likely than $\mathrm{Pt}_{\mathrm{C}_{\alpha}} @ z_{1}$.

\subsection{Pt single-atom adsorption on $a$ GNRs}

We investigate the Pt single-atom adsorption at the edge of mono- and di-hydrogenated $a$ GNRs. We do not consider nonhydrogenated $a \mathrm{GNRs}$ as the non-hydrogenated structures are unstable as observed in the case of $z$ GNR. We consider the following structures: Pt adsorbed at short-bridge (SB) and longbridge (LB) sites (Fig. 8(a) and (b), respectively); substitutional Pt with single C atom respectively (Fig. 8(c)-(e)); substitutional Pt with two C atoms (Fig. 8(f)-(i)). We construct similar structures for dihydrogenated $a$ GNR (Fig. 9). The structures are fully optimized and the Gibbs free energies are calculated according to eqn (4). The phase diagram for $a$ GNR is shown in Fig. 10, and

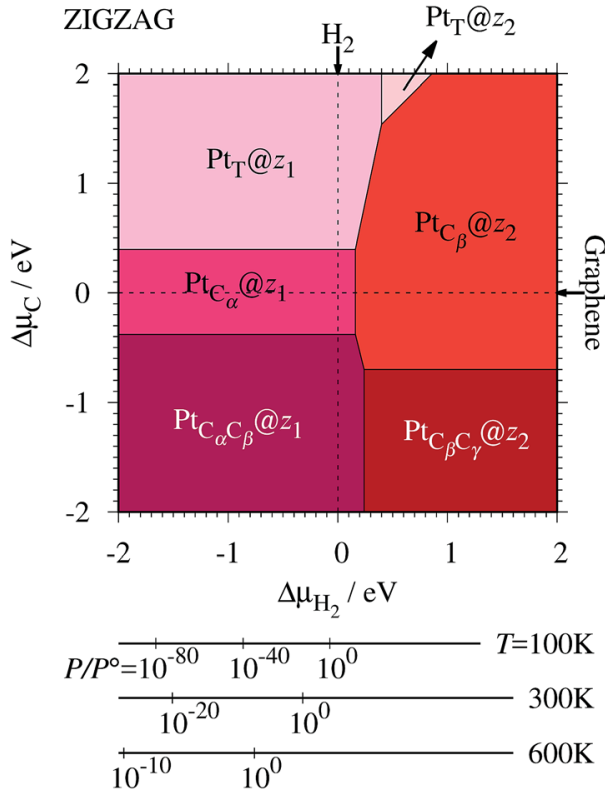

Fig. 7 The Gibbs free energy for Pt single-atom adsorption at the edge of $z$ GNRs as a function of $\mathrm{C}$ and $\mathrm{H}_{2}$ chemical potentials. $\Delta \mu_{\mathrm{C}}=\mu_{\mathrm{C}}$ - $E_{\mathrm{GR}}$ and $\Delta \mu_{\mathrm{H}_{2}}=\mu_{\mathrm{H}_{2}}-E_{\mathrm{H}_{2}}$ are $\mathrm{C}$ and $\mathrm{H}_{2}$ chemical potentials referenced to the total energies of gas-phase $\mathrm{H}_{2}$ and graphene, respectively. The bottom axes show the corresponding $\mathrm{H}_{2}$ chemical potentials at the absolute temperature $T$ and partial pressure $P$ (with $P^{\circ}$ $=1 \mathrm{~atm}), \mu_{\mathrm{H}_{2}}=H^{\circ}(T)-H^{\circ}(0)-T S^{\circ}(T)+k_{\mathrm{B}} T \ln \left(P / P^{\circ}\right)$, where the enthalpy $H^{\circ}$ and the entropy $S^{\circ}$ are obtained from ref. 66 .

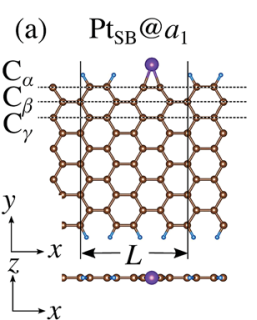

(d)

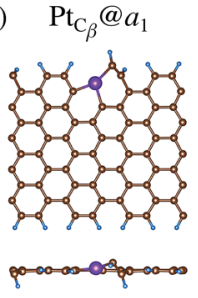

(g) $\quad \mathrm{Pt}_{\mathrm{C}_{\alpha} \mathrm{C}_{\beta}} @ a_{1}$

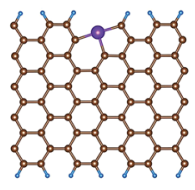

$\infty-\infty-\infty-\infty-\infty-\infty-\infty-\infty$ (b) $\mathrm{Pt}_{\mathrm{LB}} @ a_{1}$

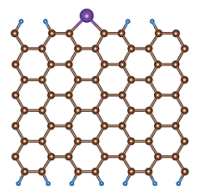

(e) $\mathrm{Pt}_{\mathrm{C}_{\gamma}} @ a_{1}$

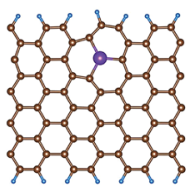

(h) $\mathrm{Pt}_{\left(\mathrm{C}_{\beta}\right)_{2}} @ a_{1}$

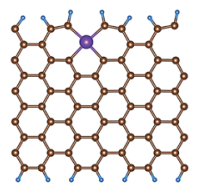

(c) $\mathrm{Pt}_{\mathrm{C}_{\alpha}} @ a_{1}$

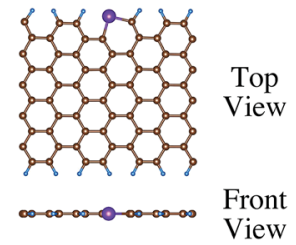

(f) $\mathrm{Pt}_{\left(\mathrm{C}_{\alpha}\right)_{2}} @ a_{1}$

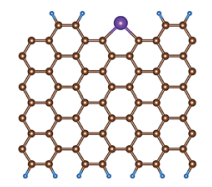

(i) $\mathrm{Pt}_{\mathrm{C}_{\beta} \mathrm{C}_{\gamma}} @ a_{1}$

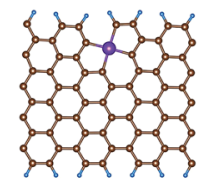

Fig. 8 Optimized structures of Pt single-atom adsorption at the edges of mono-hydrogenated aGNRs. Purple, brown, and light blue spheres represent $\mathrm{Pt}, \mathrm{C}$, and $\mathrm{H}$ atoms, respectively. The first, second, and third outermost $C$ atoms are denoted as $C_{\alpha}, C_{\beta}$, and $C_{\gamma}$, respectively. $L$ indicates the periodicity of the ribbons along the edge direction. 
$\Delta \Omega$ 's at $\mu_{\mathrm{C}}=E_{\mathrm{GR}}$ and $\mu_{\mathrm{H}_{2}}=E_{\mathrm{H}_{2}}$ for different adsorption configurations are summarized in Table 3. We find that $\mathrm{Pt}_{\mathrm{C}_{\alpha}} @ z_{2}$ is the most stable at $\mu_{\mathrm{C}}=E_{\mathrm{GR}}$ and $\mu_{\mathrm{H}_{2}}=E_{\mathrm{H}_{2}}$ with reasonable CLS, suggesting that this structure is the most likely candidate under these conditions. Furthermore, the absolute value of calculated $\Delta \Omega$ is much larger than that of the most stable $z \mathrm{GNR}$, suggesting that $\mathrm{Pt}$ adsorbed at $a \mathrm{GNR}$ is thermodynamically more stable than $\mathrm{Pt}$ adsorbed at $z \mathrm{GNR}$. We also find that $\mathrm{Pt}_{\mathrm{C}_{\beta}} @ a_{1}, \mathrm{Pt}_{\mathrm{C}_{\gamma}} @ a_{1}$, and $\mathrm{Pt}_{\mathrm{C}_{\gamma}} @ a_{2}$ show relatively large positive CLSs. However, they show large positive $\Delta \Omega$ at $\mu_{\mathrm{C}}=E_{\mathrm{GR}}$ and $\mu_{\mathrm{H}_{2}}$ $=E_{\mathrm{H}_{2}}$ and therefore appear in the phase diagram (Fig. 10). In particular the latter two show large positive $\Delta \Omega$ and are less likely.

\subsection{Impact of the substrate}

Here we examine the effect of the substrate, as in the experiment, ${ }^{42}$ Pt single-atom adsorption has been observed at the step edge of graphite, and the graphene underneath may play some role. We perform the structural optimization with the graphene substrate and calculate the CLS of Pt single-atom at the edge of $z$ GNR. We adopt the adsorption structures with $z_{1}$ and introduced graphene underneath as shown in Fig. 11. We optimize the structures and calculate the binding energy defined by

$$
E_{\mathrm{b}}=E_{\mathrm{Pt}} @_{z \mathrm{GNR}}-E_{\mathrm{ref}}-E_{\mathrm{Pt}}-\Delta N_{\mathrm{C}} E_{\mathrm{GR}}-\frac{\Delta N_{\mathrm{H}}}{2} E_{\mathrm{H}_{2}},
$$

where $E_{\mathrm{Pt} @ z \mathrm{GNR}}$ is the total energies of Pt adsorbed at $z \mathrm{GNR}$ (with and without the graphene substrate) and $E_{\text {ref }}$ is the total

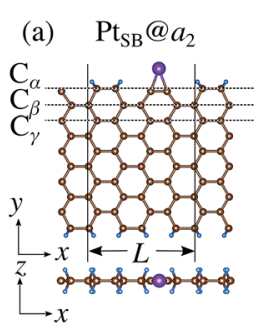

(d)

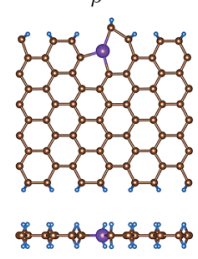

(g) $\mathrm{Pt}_{\mathrm{C}_{\alpha} \mathrm{C}_{\beta}} @ a_{2}$
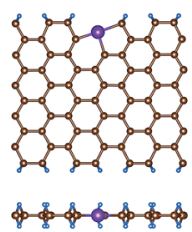

(b) $\mathrm{Pt}_{\mathrm{LB}} @ a_{2}$

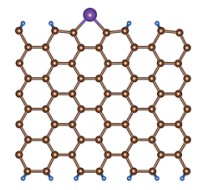

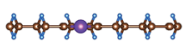

(e) $\mathrm{Pt}_{\mathrm{C}_{\gamma}} @ a_{2}$

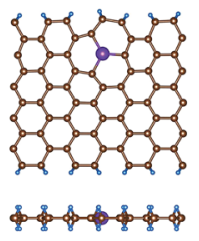

(h) $\mathrm{Pt}_{\left(\mathrm{C}_{\beta}\right)_{2}} @ a_{2}$

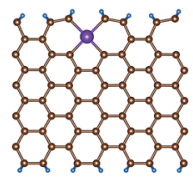

क- क- (c) $\mathrm{Pt}_{\mathrm{C}_{\alpha}} @ a_{2}$

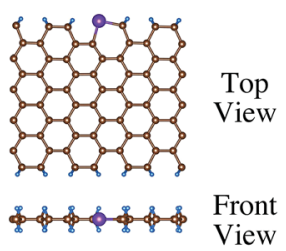

(f) $\mathrm{Pt}_{\left(\mathrm{C}_{\alpha}\right)_{2}} @ a_{2}$

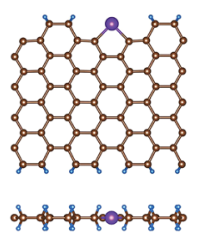

(i) $\mathrm{Pt}_{\mathrm{C}_{\beta} \mathrm{C}_{\gamma}} @ a_{2}$

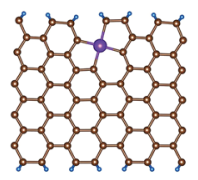

कै-क्षेक्षे
Fig. 9 Optimized structures of Pt single-atom adsorption at the edges of di-hydrogenated aGNRs. Purple, brown, and light blue spheres represent $\mathrm{Pt}, \mathrm{C}$, and $\mathrm{H}$ atoms, respectively. The first, second, and third outermost $C$ atoms are denoted as $C_{\alpha}, C_{\beta}$, and $C_{\gamma}$, respectively. $L$ indicates the periodicity of the ribbons along the edge direction.

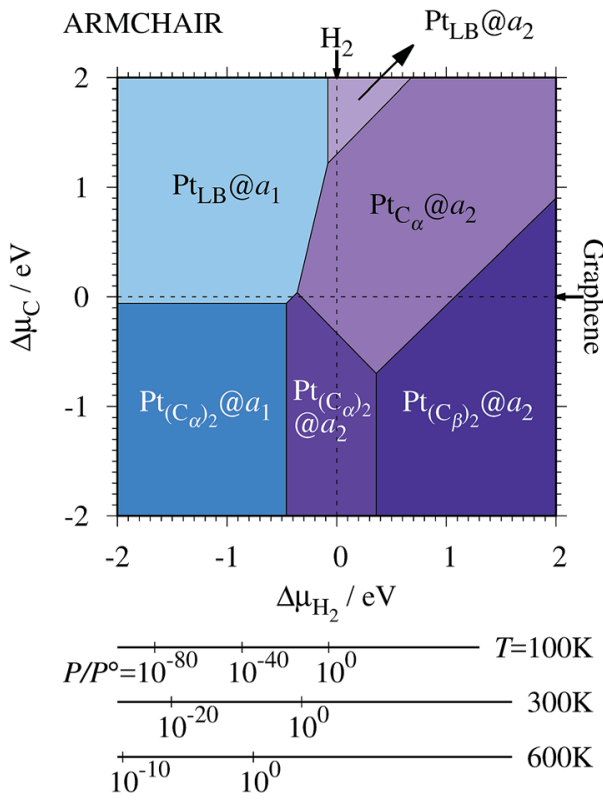

Fig. 10 The Gibbs free energy for Pt single-atom adsorption at the edges of aGNRs as a function of $\mathrm{C}$ and $\mathrm{H}_{2}$ chemical potentials. $\Delta \mu_{\mathrm{C}}=$ $\mu_{\mathrm{C}}-E_{\mathrm{GR}}$ and $\Delta \mu_{\mathrm{H}_{2}}=\mu_{\mathrm{H}_{2}}-E_{\mathrm{H}_{2}}$ are $\mathrm{C}$ and $\mathrm{H}_{2}$ chemical potentials referenced to the total energies of gas-phase $\mathrm{H}_{2}$ and graphene, respectively. The bottom axes show the corresponding $\mathrm{H}_{2}$ chemical potentials at the absolute temperature $T$ and partial pressure $P$ (with $P^{\circ}$ $=1 \mathrm{~atm}), \mu_{\mathrm{H}_{2}}=H^{\circ}(T)-H^{\circ}(0)-T S^{\circ}(T)+k_{\mathrm{B}} T \ln \left(P / P^{\circ}\right)$, where the enthalpy $H^{\circ}$ and the entropy $S^{\circ}$ are obtained from ref. 66 .

energy of the reference system, where we use perfect $z_{1}$ and perfect $z_{1}$ on graphene $\left(z_{1} / \mathrm{GR}\right)$ as references for the binding energies for a Pt single-atom at $z_{1}$ and at $z_{1} / \mathrm{GR}$, respectively. Calculated binding energies and corresponding CLSs are summarized in Table 4 . We can see that the difference of the binding energies with and without the substrate is insignificant, and in most cases, the substrate plays a role in stabilizing the Pt single-atom adsorption, except for $\mathrm{Pt}_{\mathrm{C}_{\beta}} @ z_{1}$. The most stable adsorption structure $\left(\mathrm{Pt}_{\mathrm{C}_{\alpha}} @ z_{1}\right)$ is unchanged and the binding energy difference is $0.16 \mathrm{eV}$ upon inclusion of the substrate. The substrate does have an insignificant impact on

Table 3 The Gibbs free energy $(\Delta \Omega)$ for Pt atom adsorption at aGNRs at $\mu_{\mathrm{C}}=E_{\mathrm{GR}}$ and $\mu_{\mathrm{H}}=E_{\mathrm{H}_{2}}$ and the corresponding core level shift (CLS)

\begin{tabular}{lllllr}
\hline & \multicolumn{2}{c}{$\Delta \Omega\left(E_{\mathrm{GR}}, E_{\mathrm{H}_{2}}\right) / \mathrm{eV}$} & & \multicolumn{2}{c}{$\mathrm{CLS} / \mathrm{eV}$} \\
\cline { 2 - 3 } \cline { 5 - 6 } & $a_{1}$ & $a_{2}$ & & $a_{1}$ & $a_{2}$ \\
\hline $\mathrm{Pt}_{\mathrm{SB}}$ & +0.67 & -0.32 & & +0.63 & +0.60 \\
$\mathrm{Pt}_{\mathrm{LB}}$ & -0.69 & -0.94 & & +0.44 & +0.77 \\
$\mathrm{Pt}_{\mathrm{C}_{\alpha}}$ & -0.35 & -2.24 & & +1.33 & +1.33 \\
$\mathrm{Pt}_{\mathrm{C}_{\beta}}$ & +0.91 & -0.02 & & +1.56 & +0.82 \\
$\mathrm{Pt}_{\mathrm{C}_{\gamma}}$ & +4.15 & +3.00 & & +2.36 & +2.33 \\
$\mathrm{Pt}_{\left(\mathrm{C}_{\alpha}\right)_{2}}$ & -0.57 & -1.90 & & +0.17 & +0.51 \\
$\mathrm{Pt}_{\mathrm{C}_{\alpha} \mathrm{C}_{\beta}}$ & +0.33 & -1.40 & & +0.53 & +0.77 \\
$\mathrm{Pt}_{\left(\mathrm{C}_{\beta}\right)_{2}}$ & +0.51 & -1.17 & & -0.11 & -0.74 \\
$\mathrm{Pt}_{\mathrm{C}_{\beta} \mathrm{C}_{\gamma}}$ & +1.48 & -0.44 & & +0.05 & -0.03 \\
$\mathrm{Expt}$ & - & - & & \multicolumn{2}{c}{$+2.0 \pm 0.4^{a}$}
\end{tabular}

${ }^{a}$ Taken from ref. 42 . 


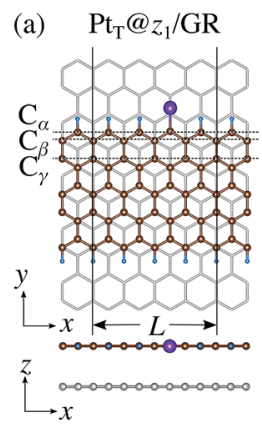

(d)

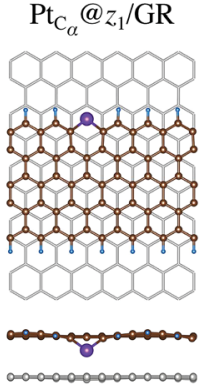

(g) $\mathrm{Pt}_{\mathrm{C}_{\alpha}} V_{\mathrm{C}_{\alpha}} @ z_{1} / \mathrm{GR}$
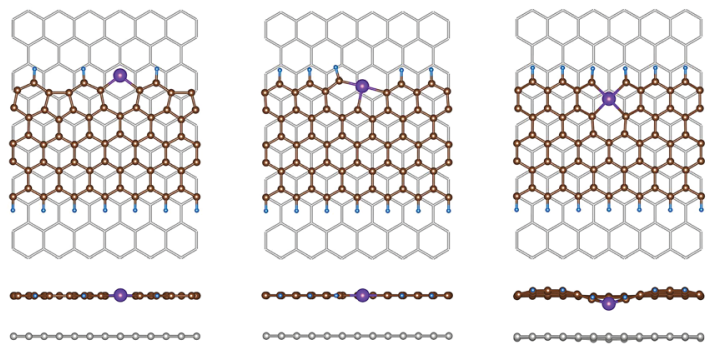

(h) $\mathrm{Pt}_{\mathrm{C}_{\alpha} \mathrm{C}_{\beta}} @ z_{1} / \mathrm{GR}$

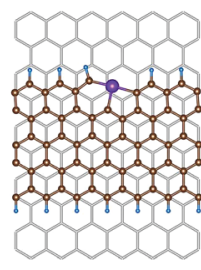

(i) $\mathrm{Pt}_{\mathrm{C}_{\beta} \mathrm{C}_{\gamma}} @ z_{1} / \mathrm{GR}$

(c) $\mathrm{Pt}_{\mathrm{LB}} @ z(57)_{1} / \mathrm{GR}$

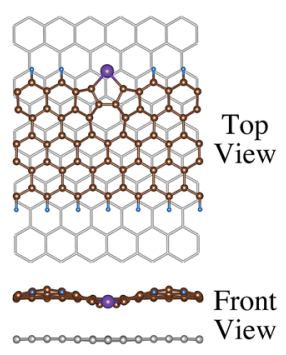

(e) $\mathrm{Pt}_{\mathrm{C}_{\beta}} @ z_{1} / \mathrm{GR}$

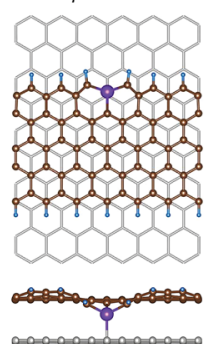

(f) $\mathrm{Pt}_{\left(\mathrm{C}_{\alpha}\right)_{2}} @ z_{1} / \mathrm{GR}$

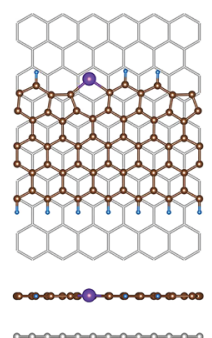

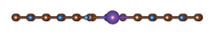

Table 4 The binding energy $\left(E_{\mathrm{b}}\right)$ and the core level shift (CLS) of Pt single-atom adsorption at the edges of $z_{1}$ with and without the substrate graphene (GR)

\begin{tabular}{|c|c|c|c|c|}
\hline & \multicolumn{2}{|c|}{$E_{\mathrm{b}} / \mathrm{eV}$} & \multicolumn{2}{|c|}{ CLS/eV } \\
\hline & w/o GR & w/GR & w/o GR & w/GR \\
\hline $\mathrm{Pt}_{\mathrm{T}} @ z_{1}$ & -2.19 & -2.32 & -0.93 & -0.94 \\
\hline $\mathrm{Pt}_{\mathrm{B}} @ z_{1}$ & -0.77 & -0.88 & +0.35 & +0.34 \\
\hline $\mathrm{Pt}_{\mathrm{LB}} @ z(57)_{1}$ & +1.55 & +1.70 & +0.44 & +0.51 \\
\hline $\mathrm{Pt}_{\mathrm{C}_{\alpha}} @ z_{1}$ & -2.58 & -2.74 & +1.43 & +1.24 \\
\hline $\mathrm{Pt}_{\mathrm{C}_{\beta}} @ z_{1}$ & -0.44 & -0.33 & +1.37 & +1.99 \\
\hline $\operatorname{Pt}\left(\mathbf{C}_{\alpha}\right) @ z_{1}$ & -0.54 & -0.58 & +0.24 & +0.21 \\
\hline $\mathrm{Pt}_{\mathrm{C}_{\alpha}} V_{\mathrm{C}_{\alpha}} @ z_{1}$ & -0.32 & -0.36 & +0.54 & -0.50 \\
\hline $\mathrm{Pt}_{\mathrm{C}_{\alpha} \mathrm{C}_{\beta}} @ z_{1}$ & -2.19 & -2.17 & +0.81 & +0.81 \\
\hline $\mathrm{Pt}_{\mathrm{C}_{\beta} \mathrm{C}_{\gamma}} @ z_{1}$ & -0.22 & -0.01 & +0.82 & +0.78 \\
\hline Expt & - & - & \multicolumn{2}{|c|}{$+2.0 \pm 0.4^{a}$} \\
\hline
\end{tabular}

${ }^{a}$ Taken from ref. 42 .

that the total energy of the most stable form of GNR at a given $\mu_{\mathrm{H}_{2}}$ is used as a reference $\left(E_{z(a) \mathrm{GNR}}\right.$, see also Fig. 2). $E_{\mathrm{b}}$ is an indicator of the strength of Pt single-atom adsorption and can be used to discuss the stability of Pt when both $z \mathrm{GNR}$ and $a \mathrm{GNR}$ are present. Calculated $E_{\mathrm{b}}$ 's as a function of $\mu_{\mathrm{C}}$ and $\mu_{\mathrm{H}_{2}}$ (phase diagram) are shown in Fig. 12 and those at $\mu_{\mathrm{C}}=E_{\mathrm{GR}}$ and $\mu_{\mathrm{H}_{2}}=$ $E_{\mathrm{H}_{2}}$ are summarized in Table 5. We find that although $a \mathrm{GNRs}$ and Pt adsorbed $a \mathrm{GNRs}$ are thermodynamically more stable than $z$ GNRs in terms of the Gibbs free energy (see Tables 3 and 2 ), Pt adsorption at the edges of $z$ GNRs is more favorable. This suggests that when armchair and zigzag edges coexist, single Pt atoms prefer to adsorb at the zigzag edge. At around $\mu_{\mathrm{C}}=E_{\mathrm{GR}}$,

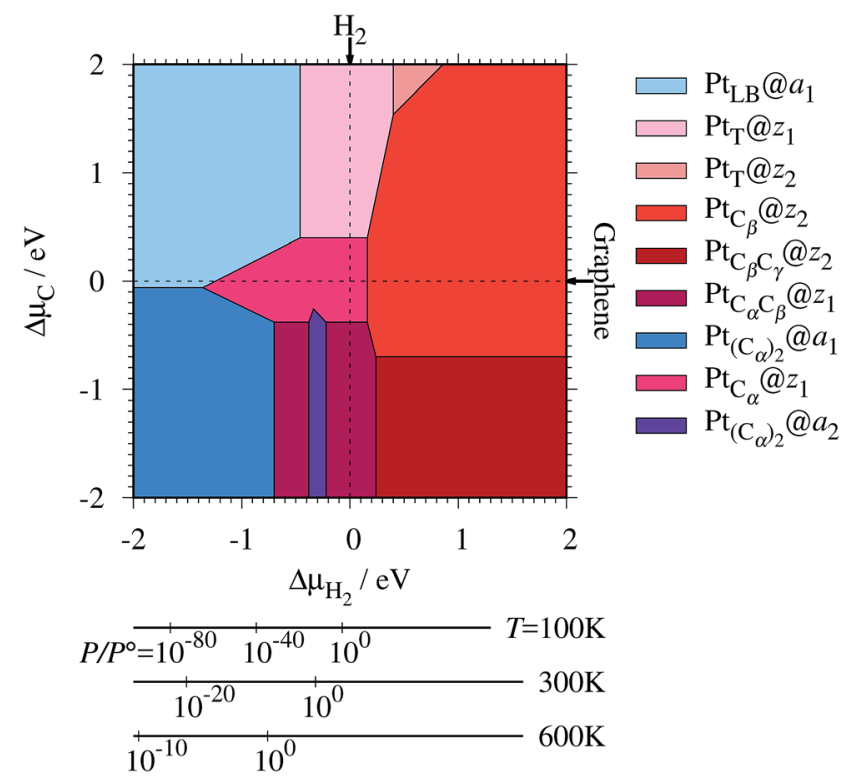

Fig. 12 The binding energy for Pt single-atom adsorption at the edge of GNRs as a function of $\mathrm{C}$ and $\mathrm{H}_{2}$ chemical potentials. The chemical potentials of $\mathrm{C}\left(\Delta \mu_{\mathrm{C}}\right)$ and $\mathrm{H}_{2}\left(\Delta \mu_{\mathrm{H}_{2}}\right)$ are referenced to the total energies of graphene and gas-phase $\mathrm{H}_{2}$ molecule at $0 \mathrm{~K}$, respectively. The bottom axes show the corresponding $\mathrm{H}_{2}$ chemical potentials at the absolute temperature $T$ and partial pressure $P$ (with $P^{\circ}=1 \mathrm{~atm}$ ), $\mu_{\mathrm{H}_{2}}=$ $H^{\circ}(T)-H^{\circ}(0)-T S^{\circ}(T)+k_{\mathrm{B}} T \ln \left(P / P^{\circ}\right)$, where the enthalpy $H^{\circ}$ and the entropy $S^{\circ}$ are obtained from ref. 66. where $E_{\mathrm{Pt} @ z(a) \mathrm{GNR}}$ and $E_{z(a) \mathrm{GNR}}$ are total energies of Pt adsorbed $z \mathrm{GNR}(a \mathrm{GNR})$ and pristine $z \mathrm{GNR}(a \mathrm{GNR})$, respectively. We note
Fig. 11 Optimized structures of Pt single-atom adsorption at the edges of mono-hydrogenated $z$ GNRs with a graphene substrate. Purple, brown, and light blue spheres represent $\mathrm{Pt}, \mathrm{C}$, and $\mathrm{H}$ atoms,

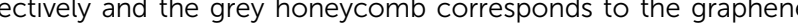
$C_{\alpha}, C_{\beta}$, and $C_{\gamma}$, respectively. $L$ indicates the periodicity of the ribbons along the edge direction.

the calculated core level shift, especially for the most stable $\mathrm{Pt}_{\mathrm{C}_{\alpha}} @ z_{1}$. The change is $-0.19 \mathrm{eV}$ and the conclusion is unaltered.

\section{Discussion} and $a \mathrm{GNR}$ on the same footing, and investigate the favorable adsorption site when both edges are exposed. For this purpose,

$$
\begin{aligned}
E_{\mathrm{b}}\left(\mu_{\mathrm{C}}, \mu_{\mathrm{H}_{2}}\right)= & E_{\mathrm{Pt}} @_{z(a) \mathrm{GNR}}-E_{z(a) \mathrm{GNR}}-E_{\mathrm{Pt}}-\Delta N_{\mathrm{C}} \mu_{\mathrm{C}} \\
& -\frac{\Delta N_{\mathrm{H}}}{2} \mu_{\mathrm{H}_{2}},
\end{aligned}
$$


Table 5 The binding energies $\left(E_{\mathrm{b}}\right)$ of the favorable Pt single-atom adsorption configurations at $\mu_{\mathrm{C}}=E_{\mathrm{GR}}$ and $\mu_{\mathrm{H}}=E_{\mathrm{H}_{2}}$

\begin{tabular}{lc}
\hline Configuration & \\
\hline $\mathrm{Pt}_{\mathrm{T}} @ z_{1}$ & $E_{\mathrm{b}} / \mathrm{eV}$ \\
$\mathrm{Pt}_{\mathrm{C}_{\alpha}} @ z_{1}$ & -2.19 \\
$\mathrm{Pt}_{\mathrm{C}_{\alpha} \mathrm{C}_{\beta}} @ z_{1}$ & -2.58 \\
$\mathrm{Pt}_{\mathrm{T}} @ z_{2}$ & -2.19 \\
$\mathrm{Pt}_{\mathrm{C}_{\beta}} @ z_{2}$ & -0.72 \\
$\mathrm{Pt}_{\mathrm{C}_{\beta} \mathrm{C}_{\gamma} @ z_{2}}$ & -1.82 \\
$\mathrm{Pt}_{\mathrm{LB}_{\mathrm{B}}} @ a_{1}$ & -1.13 \\
$\mathrm{Pt}_{\left(\mathrm{C}_{\sigma_{2}} @ a_{1}\right.}$ & -0.61 \\
$\mathrm{Pt}_{\left(\mathrm{C}_{\alpha}\right)_{2}} @ a_{2}$ & -0.49 \\
& -1.82
\end{tabular}

$\mathrm{Pt}_{\mathrm{C}_{\alpha}} @ z_{1}$ and $\mathrm{Pt}_{\mathrm{C}_{\mathrm{\beta}}} @ z_{2}$ are the most likely adsorption structures. In particular, the calculated CLS for the former agrees reasonably with the experimental one, and thus, we conclude that $\mathrm{Pt}_{\mathrm{C}_{a}} @ z_{1}$ is the most probable structure. Note that at $-0.38 \mathrm{eV} \leq$ $\Delta \mu_{\mathrm{H}_{2}} \leq-0.22 \mathrm{eV}, \operatorname{Pt}\left(\mathrm{C}_{\alpha}\right)_{2} @ a_{2}$ appears as a stable structure among $\mathrm{Pt}_{\mathrm{C}_{\alpha} \mathrm{C}_{\beta}} @ z_{1}$. This narrow $a_{2}$ region corresponds to the region where intersections of formation energies are found (Fig. 2) and the stable (unstable) phase is determined by a subtle energy balance. As a result, $a_{2}$ becomes unstable, i.e., more reactive, and $\mathrm{Pt}\left(\mathrm{C}_{\alpha}\right)_{2} @ a_{2}$ emerges as a stable adsorption structure. To determine the precise stability (boundary) when the different phases compete, however, more accurate and precise calculation of total energy is required. Nevertheless, the stability at around $\mu_{\mathrm{C}}=E_{\mathrm{GR}}$ and $\mu_{\mathrm{H}_{2}}=E_{\mathrm{H}_{2}}$ is unaffected, and we pursue this in future work. Very recently, Yamazaki et al. proposed the threefold-coordinated Pt atom at the edges of graphene flakes based on X-ray photoelectron spectroscopy and DFT calculations, ${ }^{42}$ which contradicts our finding that the thermodynamically most stable configuration $\left(\mathrm{PtCa} @ \mathrm{z}_{1}\right)$ has a twofoldcoordinated $\mathrm{Pt}$ atom. On the other hand, the metastable $\mathrm{Pt}_{\mathrm{C}_{\beta}} @ z_{1}$ in our study, which has a threefold-coordinated $\mathrm{Pt}$ atom, gives relatively large binding energy and reasonable CLS, and we do not rule out the possibility of the threefold-coordinated Pt atom at the edge at this point. To resolve the discrepancy, we may need to employ the state-of-the-art theoretical method to calculate the absolute binding energy, ${ }^{67}$ and to investigate the models for the graphene edge, because we and Yamazaki et al. use different models (GNR and graphene flakes, respectively) for the graphene edge. Nevertheless, we can conclude that the Pt single-atom adsorption takes place dominantly at the edge of graphene, and determination of a more precise position and CLS will be done in the near future.

Here, let us discuss the oxidation state of a single Pt atom, as it is crucial to the understanding of its catalytic activity. We calculated the density of states projected on the atomic orbitals of Pt for $\mathrm{Pt}_{\mathrm{C}_{\alpha}}$ @ $z_{1}$ (Fig. S3 in the ESI $\dagger$ ), which gives CLS in good agreement with the experimental value. We found that the $\mathrm{Pt}$ $\mathrm{d}$ states hybridize with the GNR state. In particular, the Pt $\mathrm{d}_{x y}$ state hybridizes strongly with the C sp state and forms a fully unoccupied antibonding state, resulting in the formal oxidation state of $2+$. We also performed the Bader charge analysis (Table S2 in the ESI $\dagger$ ), and found that it is not straightforward to assign the oxidation state of Pt only from the Bader charge analysis, especially those at the edges, because of the strong hybridization of Pt $d$ states with GNR.

\section{Conclusions}

We present a systematic density functional theory-based thermodynamics study of Pt single-atom adsorption on graphene. We find that single Pt atoms adsorb more preferably at the graphene edge than on the bulk. Although pristine $a \mathrm{GNR}$ is thermodynamically more stable than $z$ GNR under a wide range of hydrogen pressure, single Pt atoms preferably adsorb at the edge of hydrogenated $z \mathrm{GNR}$. The calculated core level shifts for the stable structures are in reasonable agreement with the experiment, supporting our findings. Our study will serve as a basis for further investigation of the catalytic activity of singleatom catalysts based on single Pt atoms and graphene based nanostructures.

\section{Conflicts of interest}

There are no conflicts to declare.

\section{Acknowledgements}

We thank Professor Kazutoshi Gohara for providing ref. 42 prior to publication. This work was partially supported by the Advanced Catalytic Transformation Program for Carbon Utilization (ACT-C) of the Japan Science and Technology Agency, by Grants-in-Aid for Scientific Research on Innovative Areas 3D Active-Site Science (JP26105010, JP26105011, and JP15K21719) from the Japan Society for the Promotion of Science, and by the Elements Strategy Initiative for Catalysts and Batteries supported by the Ministry of Education, Culture, Sports, Science, and Technology, Japan. S. A. W. acknowledges financial support by the Marubun Research Promotion Foundation and WCU-ITB Sandwich Program. Numerical calculations were performed by using the computer facilities at the Institute for Solid State Physics, University of Tokyo, at the HPCI systems provided by Nagoya University, at the University of Tokyo, and at Tohoku University through the HPCI System Research Project (Project ID: hp130112, hp140166, and hp150201).

\section{References}

1 P. Costamagna and S. Srinivasan, J. Power Sources, 2001, 102, 242-252.

2 H. Liu, C. Song, L. Zhang, J. Zhang, H. Wang and D. P. Wilkinson, J. Power Sources, 2006, 155, 95-110.

3 H. A. Gasteiger, N. M. Marković, P. N. Ross and E. J. Cairns, Electrochim. Acta, 1994, 39, 1825-1832.

4 A. Kabbabi, R. Faure, R. Durand, B. Beden, F. Hahn, J. M. Leger and C. Lamy, J. Electroanal. Chem., 1998, 444, 41-53.

5 B. N. Grgur, N. M. Marković and P. N. Ross, J. Phys. Chem. B, 1998, 102, 2494-2501.

6 H. A. Gasteiger, N. M. Marković and P. N. Ross, Catal. Lett., 1996, 36, 1-8. 
7 M. Watanabe, Y. Zhu and H. Uchida, J. Phys. Chem. B, 2000, 104, 1762-1768.

8 H. Igarashi, T. Fujino, Y. Zhu, H. Uchida and M. Watanabe, Phys. Chem. Chem. Phys., 2001, 3, 306-314.

9 J. Lai, R. Luque and G. Xu, ChemCatChem, 2015, 7, 32063228.

10 T. Ozaki, J.-I. andi Anahara, N. Kimura and A. Oya, Carbon, 2006, 44, 3358-3361.

11 J.-I. Ozaki, N. Kimura, T. Anahara and A. Oya, Carbon, 2007, 45, 1847-1853.

12 P. H. Matter, E. Wang, M. Arias, E. J. Biddinger and U. S. Ozkan, J. Mol. Catal. A: Chem., 2007, 264, 73-81.

13 X. Huang, Y. Wang, W. Li and Y. Hou, Sci. China: Chem., 2017, 60, 1494-1507.

14 F. Rodríguez-Reinoso, I. Rodríguez-Ramos, C. MorenoCastilla, A. Guerrero-Ruiz and J. D. López-González, J. Catal., 1986, 99, 171-183.

15 T. Yoshitake, Y. Shimakawa, S. Kuroshima, H. Kimura, T. Ichihashi, Y. Kubo, D. Kasuya, K. Takahashi, F. Kokai, M. Yudasaka and S. Iijima, Phys. Rev. B: Condens. Matter Mater. Phys., 2002, 323, 124-126.

16 E. Yoo, T. Okata, T. Akita, M. Kohyama, J. Nakamura and I. Honma, Nano Lett., 2009, 9, 2255-2259.

17 J. Oh, T. Kondo, D. Hatake, Y. Iwasaki, Y. Honma, Y. Suda, D. Sekiba, H. Kudo and J. Nakamura, J. Phys. Chem. Lett., 2010, 1, 463-466.

18 J. Nakamura, Carbon, 2015, 85, 443-444.

19 K. Ding, A. Gulec, A. M. Johnson, N. M. Schweitzer, G. D. Stucky, L. D. Marks and P. C. Stair, Science, 2015, 350, 189-192.

20 L. Xin, F. Yang, S. Rasouli, Y. Qiu, Z.-F. Li, A. Uzunoglu, C.-J. Sun, Y. Liu, P. Ferreira, W. Li, Y. Ren, L. A. Stanciu and J. Xie, ACS Catal., 2016, 6, 2642-2653.

21 H. C. Dam, N. T. Cuong, N. A. Tuan, Y.-T. Kim, H. T. Bao, T. Mitani, T. Ozaki and H. Nagao, Chem. Phys. Lett., 2006, 432, 213-217.

22 H. C. Dam, N. T. Cuong, A. Sugiyama, T. Ozaki, A. Fujiwara, T. Mitani and S. Okada, Phys. Rev. B: Condens. Matter Mater. Phys., 2009, 79, 115426.

23 K. Okazaki-Maeda, Y. Morikawa, S. Tanaka and M. Kohyama, Surf. Sci., 2010, 604, 144-154.

24 T. L. Pham, P. V. Dung, A. Sugiyama, N. D. Duc, T. Shimoda, A. Fujiwara and D. H. Chi, Comput. Mater. Sci., 2010, 49, S15S20.

25 M. Zhou, A. Zhang, Z. Dai, C. Zhang and Y. P. Feng, J. Chem. Phys., 2010, 132, 194704.

26 Y. Tang, Z. Yang and X. Dai, Phys. Chem. Chem. Phys., 2012, 14, 16566-16572.

27 I. Fampiou and A. Ramasubramaniam, J. Phys. Chem. C, 2012, 116, 6543-6555.

28 K. E. Hayes and H.-S. Lee, Chem. Phys., 2012, 393, 96-106.

29 J. Russell, P. Zapol, P. Král and L. A. Curtiss, Chem. Phys. Lett., 2012, 536, 9-13.

30 A. Chutia, I. Hamada and M. Tokuyama, Surf. Sci., 2014, 628, 116-125.

31 R. J. Gasper and A. Ramasubramaniam, J. Phys. Chem. C, 2016, 120, 17408-17417.
32 H. Shi, S. M. Auerbach and A. Ramasubramaniam, J. Phys. Chem. C, 2016, 120, 11899-11909.

33 C. R. C. Rêgo, P. Tereshchuk, L. N. Oliveira and J. L. F. Da Silva, Phys. Rev. B: Condens. Matter Mater. Phys., 2017, 95, 235422.

34 M. Mahmoodinia, P.-O. Åstrand and D. Chen, J. Phys. Chem. C, 2017, 121, 20802-20812.

35 S. Sun, G. Zhang, N. Gauquelin, N. Chen, J. Zhou, S. Yang, W. Chen, X. Meng, D. Geng, M. N. Banis, R. Li, S. Ye, S. Knights, G. A. Botton, T.-K. Sham and X. Sun, Sci. Rep., 2013, 3, 1775.

36 N. Cheng, S. Stambula, D. Wang, M. N. Banis, J. Liu, A. Riese, B. Xiao, R. Li, T.-K. Sham, L.-M. Liu, G. A. Botton and X. Sun, Nat. Commun., 2016, 7, 13638.

37 S. Back, J. Lim, N.-Y. Kim, Y.-H. Kim and Y. Jung, Chem. Sci., 2017, 8, 1090-1096.

38 K.-J. Kong, Y. Choi, B.-H. Ryu, J.-O. Lee and H. Chang, Mater. Sci. Eng., C, 2006, 26, 1207-1210.

39 H. Wang, K. Li, Y. Cheng, Q. Wang, Y. Yao, U. Schwingenschlögl, X. Zhang and W. Yang, Nanoscale, 2012, 4, 2920-2925.

40 J. Zhao, Q. Deng, S. M. Avdoshenko, L. Fu, J. Eckert and M. H. Rümmeli, Proc. Natl. Acad. Sci. U. S. A., 2014, 111, 15641-15646.

41 E. Kano, A. Hashimoto and M. Takeguchi, Appl. Phys. Express, 2017, 10, 025104.

42 K. Yamazaki, Y. Maehara, C.-C. Lee, J. Yoshinobu, T. Ozaki and K. Gohara, J. Phys. Chem. C, 2018, 122, 27292-27300.

43 A. Hashimoto and M. Takeguchi, J. Electron Microsc., 2012, 61, 409-413.

44 T. Tomai, Y. Kawaguchi, S. Mitani and I. Honma, Electrochim. Acta, 2013, 92, 421-426.

45 Y. Shen, Z. Zhang, K. Xiao and J. Xi, Phys. Chem. Chem. Phys., 2014, 16, 21609-21614.

46 K. Reuter and M. Scheffler, Phys. Rev. B: Condens. Matter Mater. Phys., 2003, 68, 045407.

47 Y. Morikawa, K. Iwata and K. Terakura, Appl. Surf. Sci., 2001, 169-170, 11-15.

48 Y. Morikawa, H. Ishii and K. Seki, Phys. Rev. B: Condens. Matter Mater. Phys., 2004, 69, 041403.

49 D. Vanderbilt, Phys. Rev. B: Condens. Matter Mater. Phys., 1990, 41, 7892-7895.

50 I. Hamada, Phys. Rev. B: Condens. Matter Mater. Phys., 2014, 89, 121103.

51 Y. Hamamoto, I. Hamada, K. Inagaki and Y. Morikawa, Phys. Rev. B: Condens. Matter Mater. Phys., 2016, 93, 245440.

52 G. Román-Pérez and J. M. Soler, Phys. Rev. Lett., 2009, 103, 096102.

53 J. Wu and F. Gygi, J. Chem. Phys., 2012, 136, 224107.

54 J. P. Perdew, K. Burke and M. Ernzerhof, Phys. Rev. Lett., 1996, 77, 3865-3868.

55 M. Callsen and I. Hamada, Phys. Rev. B: Condens. Matter Mater. Phys., 2015, 91, 195103.

56 P. Y. Huang, C. S. Ruiz-Vargas, A. M. van der Zande, W. S. Whitney, M. P. Levendorf, J. W. Kevek, S. Garg, J. S. Alden, C. J. Hustedt, Y. Zhu, J. Park, P. L. McEuen and D. A. Muller, Nature, 2011, 469, 389. 
57 F. R. Eder, J. Kotakoski, U. Kaiser and J. C. Meyer, Sci. Rep., 2014, 4, 4060.

58 T. Wassmann, A. P. Seitsonen, A. M. Saitta, M. Lazzeri and F. Mauri, Phys. Rev. Lett., 2008, 101, 096402.

59 S. Okada and A. Oshiyama, Phys. Rev. Lett., 2001, 87, 146803. 60 H. Lee, Y.-W. Son, N. Park, S. Han and J. Yu, Phys. Rev. B: Condens. Matter Mater. Phys., 2005, 72, 174431.

61 S. A. Wella, H. Sawada, N. Kawaguchi, F. Muttaqien, K. Inagaki, I. Hamada, Y. Morikawa and Y. Hamamoto, Phys. Rev. Mater., 2017, 1, 061001.

62 Y. Baskin and L. Meyer, Phys. Rev., 1955, 100, 544.

63 E. Pehlke and M. Scheffler, Phys. Rev. Lett., 1993, 71, 23382341.
64 S. García-Gil, A. García and P. Ordejón, Eur. Phys. J. B, 2012, 85, 239.

65 F. A. Kröger and H. J. Vink, in Relations between the Concentrations of Imperfections in Crystalline Solids, ed. F. Seitz and D. Turnbull, Academic Press, 1956, vol. 3, pp. 307-435.

66 M. W. Chase Jr, C. A. Davies, J. R. Downey Jr, D. J. Frurip, R. A. McDonald and A. N. Syverud, NIST-JANAF Thermochemical Tables, J. Phys. Chem. Ref. Data, Monograph 9, 4th edn, 1998, pp. 1-1951.

67 T. Ozaki and C.-C. Lee, Phys. Rev. Lett., 2017, 118, 026401. 